\title{
Teaching Artificial Intelligence and Robotics to Undergraduate Systems Engineering Students
}

\author{
Luis Emilio Alvarez-Dionisi \\ School of Systems Engineering, \\ Polytechnic University Institute "Santiago Mariño," Barinas, Venezuela \\ Email: dr.luis.alvarez@outlook.com
}

\author{
Mitali Mittra \\ Russian Presidential Academy of National Economy and Public Administration (RANEPA), \\ Kingston/RANEPA MBA Program, Moscow, Russian Federation \\ Email: mitalim88@gmail.com \\ Rosbelia Balza \\ School of Systems Engineering, \\ Polytechnic University Institute "Santiago Mariño," Barinas, Venezuela \\ Email: rosbelia@hotmail.com
}

Received: 22 April 2017; Accepted: 13 September 2017; Published: 08 July 2019

\begin{abstract}
The skills of artificial intelligence (AI) and robotics provide a wide window of job opportunities for the following professionals: computer scientists, mechanical engineers, system engineers, computer engineers, biomedical engineers, and electrical engineers. Additionally, other professionals benefiting from AI and robotics' job opportunities are information technologists, informatic engineers, electronic engineers, data scientists, industrial engineers, big data engineers, and related specialists in the dynamic field of engineering robotics. Therefore, the purpose of this research was to study the effort of teaching AI and robotics to undergraduate systems engineering students at the Polytechnic University Institute "Santiago Mariño" in Barinas, Venezuela. Consequently, the methodology used in this academic research was the case study approach, which included three phases, namely Initiation Phase, Fieldwork Phase, and Closing Phase. In that sense, the design of research adopted in this study was based on the development of an exploratory single case study method. As a result, the Theoretical Framework created as a cornerstone of this research highlighted the following three research variables: (1) Robotic Applications, (2) Mechanics of Robotic Manipulation and Computer Vision, and (3) Object-oriented Analysis and Design (OOAD) and Object-oriented (OO) High-level Programming Languages. In conclusion, two nondirectional null hypotheses were tested, leading to the positive answers of the following research questions: (1) "Can undergraduate systems engineering students apply OOAD and OO High-level Programming Languages to analyze, design, and develop Robotic Applications?" and (2) "Can undergraduate systems engineering students use Mechanics of Robotic Manipulation and Computer
\end{abstract}

Vision to analyze, design, and develop Robotic Applications?" as stated in this case study.

Index Terms-Artificial Intelligence, Robotics, Big Data, Case Study, Universal Robots.

\section{INTRODUCTION}

In order to move forward, let's begin crafting this paper by defining the meaning of $\mathrm{AI}$ and denoting what robotics is all about.

In a nutshell, AI is "a way of making a computer, a computer-controlled robot, or software think intelligently, in the similar manner the intelligent humans think" $[1, \mathrm{p}$. $1]$.

Nonetheless, the father of AI, John McCarthy, defines $\mathrm{AI}$ as the science and engineering of creating intelligent machines, particularly smart computer programs [1].

Furthermore, AI techniques can be used to generate new ideas in the following three ways: (1) by creating innovative combinations of linked ideas, (2) by discovering the potential of theoretical spaces, and (3) by making transformations that enable the production of previously unfeasible ideas [2].

On the other hand, the idea of intelligent machines leads to the notions of robots and robotics [3].

However, the term robot is coming from the Czech language and it means enforced labor work. As a matter of fact, this word was invented by Czech writer Karel Capek in 1921 and it was introduced in his work named the Rossum's Universal Robots (R.U.R.) [4].

Additionally, Isaac Asimov further coined the term robotics [1]. In fact, robotics is the science responsible for studying robots. 
In reality, robotics is an interdisciplinary field, where several areas come together, ranging from cognitive sciences, electrical engineering, computer science, mechanical engineering, and control theory. However, bioengineering, cognitive sciences, and material physics are three innovative areas that are contributing to shaping the definition of robotics nowadays [5].

Nevertheless, robotics is a discipline based on two enables technologies [6].

(1) Telemanipulators: These are remotely controlled machines which frequently consist of an arm and a gripper.

(2) Numerical Control of Machines: These are technologies that allow controlling machines in relation to a given coordinate system. The first numerical control machine was used in 1952 at the Massachusetts Institute of Technology (MIT) and originated the first programming language for machines denominated Automatic Programmed Tools (APT).

Additionally, in order to grasp the context of robotics and the idea of a robot, it is necessary to understand the paradigm of an agent and its environment.

In that sense, an agent is denoted as an entity that can perform an activity on behalf of a user [7]. Likewise, agents have the capability to gain knowledge from the environment.

Consequently, Fig. 1 shows a representative sample of An Agent and its Environment. By the same token, a human agent has sensory organs such as ears, eyes, tongue, nose, and skin. Similarly, organs such as legs, hands, and mouth are used as effectors. In the same way, a robotic agent uses cameras, radars, and infrared range finders for sensors, and various motors for effectors.

As a result, a robot can be defined as an artificial agent that is "aimed at manipulating the objects by perceiving, picking, moving, modifying the physical properties of object, destroying it, or to have an effect thereby freeing manpower from doing repetitive functions without getting bored, distracted, or exhausted" [1, p. 47].

Robots are agents or actors that perform activities by influencing the physical world. Therefore, robots are characterized by having a close interaction with their environment. For that reason, robots have the mandate to achieve a final objective in order to accomplish a benefit for human beings.

That is why, robots are equipped with sensors with the aim to understand their environment and effectors to assert physical forces on it.

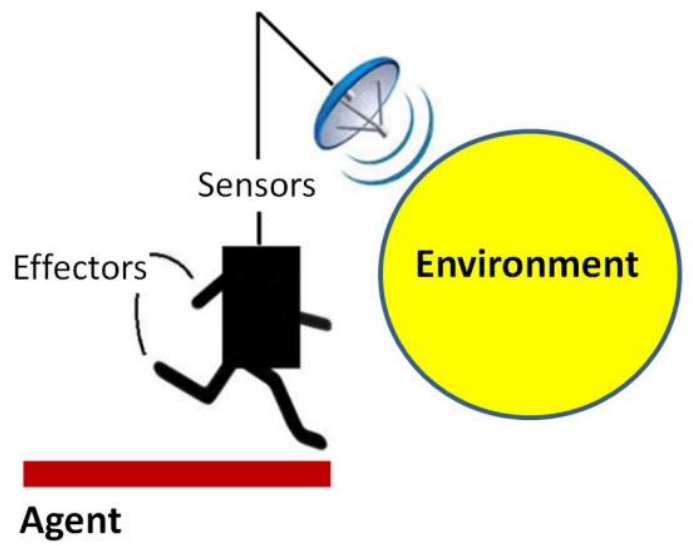

Fig.1. An Agent and its Environment (Adapted from Artificial Intelligence: Intelligence Systems [1])

According to Coppin, the main classes of agents are defined in the following paragraphs [7].

Reactive Agents: A reactive agent (also known as a reflex agent) is a production system where inputs from the environment are compared with a set of rules in order to decide which actions to take.

Interface Agents: An interface agent is typically an autonomous agent. This type of agent is capable of learning in order to perform tasks on behalf of human users. Likewise, the interface agent is able to learn by seeking advice from other agents.

Mobile Agents: A mobile agent (also known as a dynamic agent) is the one capable of "moving" from one place to another within a physical space.

Information Agents: An information agent (also known as an information-gathering agent or internet agent) is the one capable of finding, filtering, and classifying information from an immense array of sources available on the Internet.

Collaborative Agents: The collaborative agent systems are multiagent systems that represent an avenue for exploiting the combination of many agents in one system.

Additionally, with the application of state-of-the-art technologies such as machine learning, microservices, containers, big data, and deep learning, an innovative technological binomial has emerged by the name of "AIrobotics Ecosystem" where AI and robotics have been concatenated as a technological driver to provide solutions for human beings.

As a result, the field of AI-robotics Ecosystem represents a dynamic area of interest for researchers and practitioners. 
On the other hand, while robots can be categorized in many ways; in this paper, we highlight Zabala categorization of robots as follow [8].

According to Their Use: This first categorization groups the robots based on their usage.

(1) Industrial: In this classification, the robots are used as part of an industrial process.

(2) Space: In here, the robots are utilized in unexplored areas far away from their control center.

(3) Medical: This classification is used whenever the robots are used to support medical interventions.

(4) Domestic: This is where the robots are used to perform housing activities such as vacuum cleaning, dishwashing, painting, among other domestic roles.

(5) Social: These are the robots engaged to perform social activities such as movies projection, supermarket shopping, and cocktails preparation.

(6) Agriculture: In this classification, the robots are used to perform agriculture oriented supporting activities.

In addition to the above groups, we have identified a military group that addresses the application of robots in the military landscape.

Depending on the Environment Where the Activity is Performed: This second categorization portrays the robots into the following scenarios.

(1) Aquatic: This environment is characterized by having robots operating in a water-based surrounding using three-dimensional movements from the mechanic and electronic perspective.

(2) Terrestrial: These types of robots are more popular and inexpensive. They can be classified according to their mobile system as fixed, wheels, caterpillar, legs, and dragging.

(3) Airborne: This environment is characterized by having robots operating above the ground.

(4) Hybrid: These are the types of robots that are characterized by a combination of the previously highlighted environments (aquatic, terrestrial, or airborne).

According to the Location of the Robot Intelligence: This third categorization visualizes the robot's intelligence as follow.

(1) Autonomous: In this classification, the intelligence is embedded inside the robot.

(2) Automatic Control: In here, most of the intelligence is located away from the robot in a central system. The sensors can be local to the robot and they are responsible for sending the data to the central system. Thereafter, the central system sends instructions to be executed by the robot.
(3) Hybrid: This classification includes some independent robots that can be controlled by humans or a central system.

Consequently, the components of a robot are highlighted as following [1].

Power Supply: Robots are powered by batteries, hydraulic and pneumatic power sources, or solar power.

AC and DC Electric Motors: They are necessary for rotational movement.

Pneumatic Air Muscles: They provide force to the robots.

Piezo Motors and Ultrasonic Motors: They are necessary for rotational movement (normally used in industrial robots).

Muscle Wires: They supply energy to the robots.

Sensors: They provide knowledgeable information about the environment.

Additionally, computer vision is a technology of AI with which the robots can see. Therefore, computer vision plays a vital role in safety, health, security, access, and entertainment in robotics [1].

As a result, the knowledge gaining a set of education and training represents a critical success factor of the AIrobotics Ecosystem world.

For that reason, this paper was written to share the experience of teaching AI and robotics.

Accordingly, the rest of this paper is organized into the following sections: related work, describing the artificial intelligence and robotics' course, introducing the case study, resources of the course, robotic arm, theoretical framework, research questions with hypotheses, linking robotics with big data, introducing chatbots, and conclusion and recommendations.

\section{RELATED WORK}

In this section, we identified some of the fundamental research work that was previously performed on the educational AI-robotics space.

For instance, Klassner [9] conducted research on the use of LEGO Mindstorms ${ }^{\mathrm{TM}}$ as a hardware platform for integrating robotics into an AI course. In that sense, this academic experience was organized around the Russell and Norvig Agent Paradigm. Similarly, Eguchi [10] developed and delivered an educational robotics course using the LEGO Mindstorms as well. This course achieved a significant level of collaboration and cooperation from the course participants.

Likewise, Alimisis [11] from the School of Pedagogical and Technological Education in Patras, Greece, conducted an analysis of the dynamic field of educational robotics. As a result, he proposed the creation of a network of teachers and researchers in Europe dedicated to supporting the expansion of the robotics movement in education. 
On the other hand, looking at the training of engineers in robotics; Robinson [12] from the University of Plymouth, UK, discussed a strategy for developing robotics education and training for engineers.

Equally, Wong, Zink, and Koenig [13] from the University of Southern California conducted research on teaching AI and robotics using video-game technology. In this case, computer games were used as motivator factors for the students.

By the same talking, Taylor [14] from the University of Hull, UK, studied the creation of courses in robotics and advanced automation at several UK universities. Additionally, his research work described the link between industry, research, and robotics' courses.

\section{DESCRIBING THE ARTIFICIAL INTELLIGENCE AND ROBOTICS' COURSE}

This undergraduate system engineering course covered the foundation and principles of AI and robotics. In that sense, the topics included in this class were the fundamentals of AI, intelligence systems, AI research areas, mechanics of robotic manipulation, and components of robotic systems. Consequently, the primary application of this learning experience, included robotic arms, sensors, effectors, kinematics, and programming of robotic mechanisms. Similarly, the recommended background necessary to take this class included knowledge of OOAD, working knowledge of calculus, expertise in differential equations, knowledge of linear algebra, expertise in system theory, and knowledge of OO High-level Programming Languages.

\section{INTRODUCING THE CASE STUDY}

While the case study approach has been successfully used in social science [15], in this research endeavor, we used it as a research method to study the effort of teaching AI and robotics.

Likewise, we have used Alvarez-Dionisi and Tapia de Vidal's Case Studies Methodology to develop the case study of this paper as depicted in Fig. 2 [16].

Though, it is important to highlight that the case study is considered a solid research approach, which is actually used over the entire research life cycle [17]. Similarly, case studies can be analyzed and classified as explanatory, exploratory, or descriptive [15]. Additionally, they can also be structured into single, holistic, and multiple case studies $[15,17]$.

As a result, in this research project, we have adopted an exploratory single case design method.

Accordingly, the key stakeholders of this case study were five senior students coming from the undergraduate systems engineering degree program.

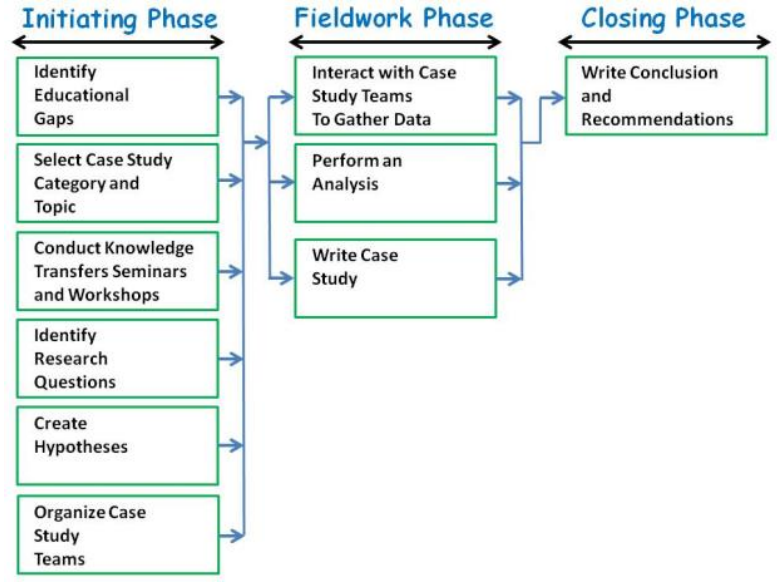

Fig.2. Case Studies Methodology [16]

In the same way, Fig. 3 shows the outcome of the evaluation of the case study's stakeholders knowledge using the following attributes.

Knowledge of OOAD: This included understanding objects and classes, encapsulation and data hiding, inheritance, message passing, polymorphism, modularity, concurrency, and persistence for the analysis \& design of the object-oriented system.

Working Knowledge of Calculus: This included limits and continuity, basic rules of derivatives, applications of the derivative, the idea of the integral, antiderivatives, summation vs. integration, indefinite integrals and substitutions, the definite integral, the fundamental theorem of calculus, applications of integration, polar coordinates, and complex numbers.

Knowledge of Mechanics of Robotic Manipulation: This had to do with robotic arms, robotic hands, rigidbody mechanics, kinetostatics of serial robots, rigid-body kinematics, kinematic decoupling, velocity of kinematic, and trajectory planning.

Expertise in Differential Equations: This included understanding first-order differential equations, linear equations of a higher order, power series methods, the Laplace transform, linear systems of differential equations, numerical methods, and nonlinear differential equations and stability.

Knowledge of Computer Vision: This had to do with geometry image features, camera models and calibration, multiple view geometry, and image segmentation.

Knowledge of Linear Algebra: This included systems of linear equations and matrices, orthogonality and least squares, determinants, Eigenvalues and Eigenvectors, Euclidean vector spaces, inner product spaces, and linear transformations.

Expertise in System Theory: This included general system theory, the organism as a physical system, open system model, the system concept of the human being, system concept in ecology, and system concept in psychology. 
Knowledge of OO High-level Programming Languages: This had to do with having experience using one or more OO High-level Programming Languages such as $\mathrm{C}++, \mathrm{C \#}$, Java, Python, or MATLAB.

\section{Evaluation}

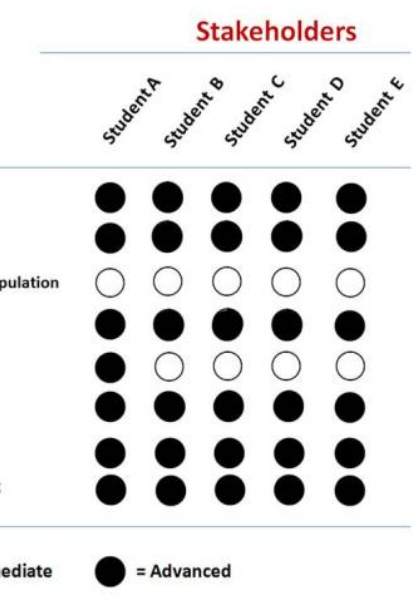

Fig.3. Evaluation of Stakeholders Knowledge

\section{RESOURCES OF THE COURSE}

During the delivery of this course, the following two groups of references were used to prepare the class.

Artificial Intelligence: This group included a collection of the following books.

(1) Artificial Intelligence: Intelligence Systems from Tutorialspoint [1].

(2) Artificial Intelligence: A Modern Approach Third Edition from Stuart J. Russell and Peter Norvig [18].

(3) Artificial Intelligence Illuminated from Ben Coppin [7].

Robotics: This group included a set of the following books.

(1) Robotics for Engineers from Yoram Korem [4].

(2) Introduction to Robotics from John J. Craig [19].

(3) Robotics Manipulation Strategies from Michael A. Peshkin [20].

As a result, the covers of the above books are depicted in the following figures: Fig. 4, Fig. 5, Fig. 6, Fig. 7, Fig. 8, and Fig. 9.

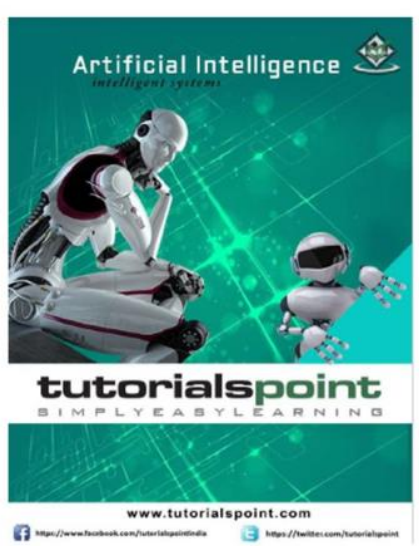

Fig.4. Artificial Intelligence: Intelligence Systems [1]

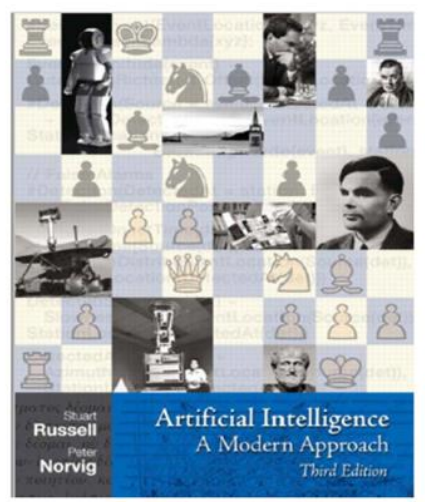

Fig.5. Artificial Intelligence: A Modern Approach Third Edition [18]

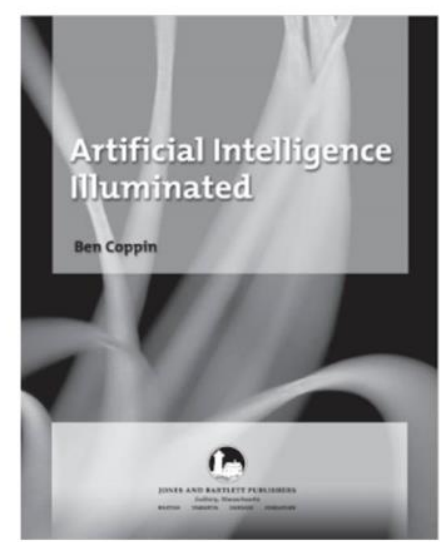

Fig.6. Artificial Intelligence Illuminated [7] 


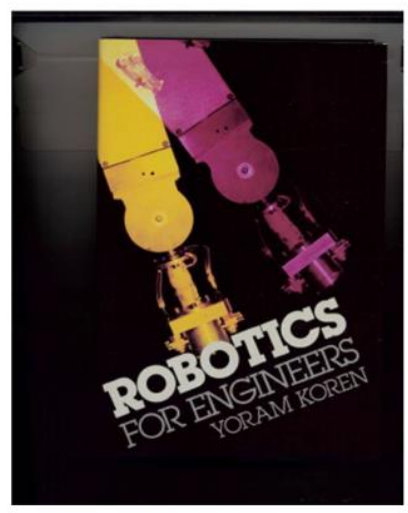

Fig.7. Robotics for Engineers [4]

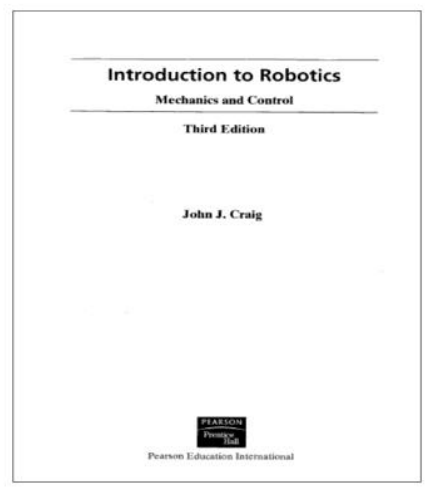

Fig.8. Introduction to Robotics [19]

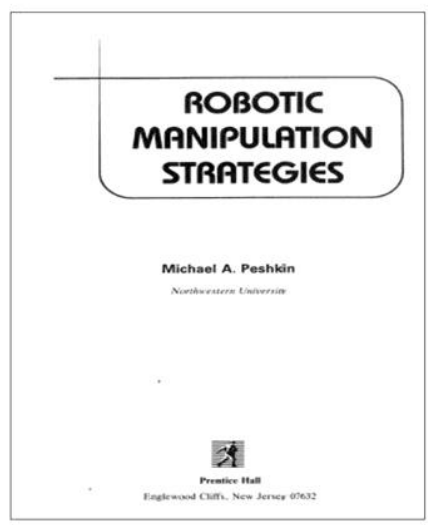

Fig.9. Robotics Manipulation Strategies [20]

Additionally, during the delivery of the course, we used the state-of-the-art online robotics training program from Universal Robots. This outstanding teaching technology allowed the systems engineering students to learn robotics in a very short time. This easy to grasp online training program was structured into the following six modules [14].

Module 1: First look - features and terminology

Module 2: How the robot works

Module 3: Setting up a tool

Module 4: Creating a program

Module 5: Interaction with external devices

Module 6: Safety settings
Consequently, Fig. 10 displays an illustration of the Universal Robots Online Training Environment.

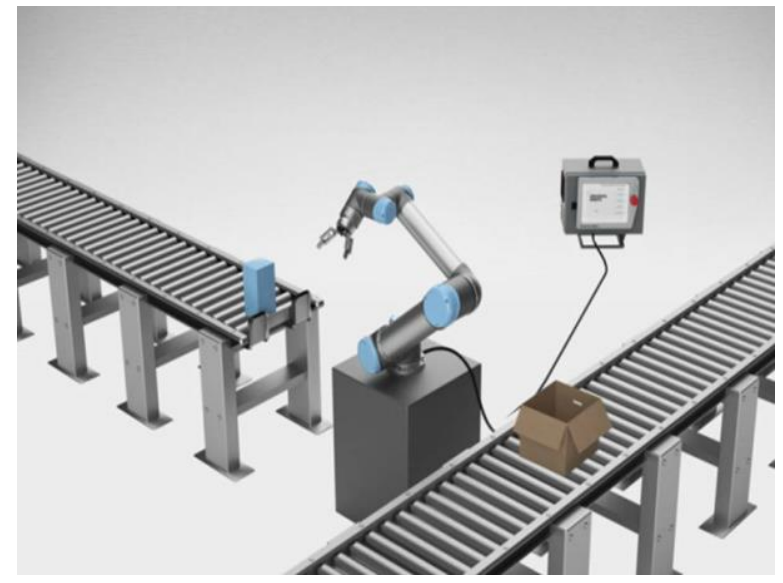

Fig.10. Universal Robots Online Training Environment [21]

\section{ROBOTIC ARM}

The robotic arm is a mechanical system resembling the human arm that is considered the simplest architecture of the robot. Generally, the robotic arm is designed with a structurally robust link coupled by either rotational or translating joints. Likewise, the robotic arm is denoted as the manipulator of the robot [22].

In Fig. 11 is depicted as an example of the robotic arm from Universal Robots Online Training Environment, which was utilized during the course of our class.

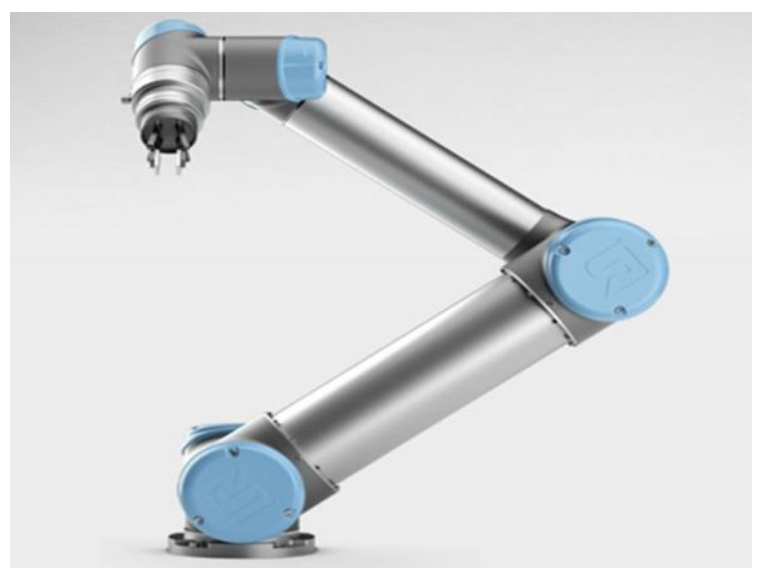

Fig.11. Universal Robots - Robotic Arm [21]

\section{THEORETICAL FRAMEWORK}

The analysis of the undergraduate course of AI and robotics at the Polytechnic University Institute "Santiago Mariño" has revealed the following two gaps: (1) there is a need from the systems engineering students point of view to integrate Robotic Applications development with OOAD and OO High-level Programming Languages and (2) there is a need from the systems engineering students perspective to integrate Robotic Applications 
development with Mechanics of Robotic Manipulation and Computer Vision.

Therefore, in order to address the above gaps, it was necessary to find answers to the following research questions.

RQ1: Can undergraduate systems engineering students apply OOAD and OO High-level Programming Languages to analyze, design, and develop Robotic Applications?

RQ2: Can undergraduate systems engineering students use Mechanics of Robotic Manipulation and Computer Vision to analyze, design, and develop Robotic Applications?

In addition to the previous research questions, we performed the following research activities: (1) define the research theoretical framework and (2) describe the research variables and hypotheses of the study.

Therefore, the Theoretical Framework of this research is depicted in Fig. 12. This figure shows the relationship of the research variables under study. In fact, it is used to hypothesize the interrelationship of such variables. As a matter of fact, in this Theoretical Framework, there are three variables: (1) Robotic Applications, (2) Mechanics of Robotic Manipulation and Computer Vision, and (3) OOAD and OO High-level Programming Languages, along with two hypotheses: $\mathrm{H} 1$ and $\mathrm{H} 2$.

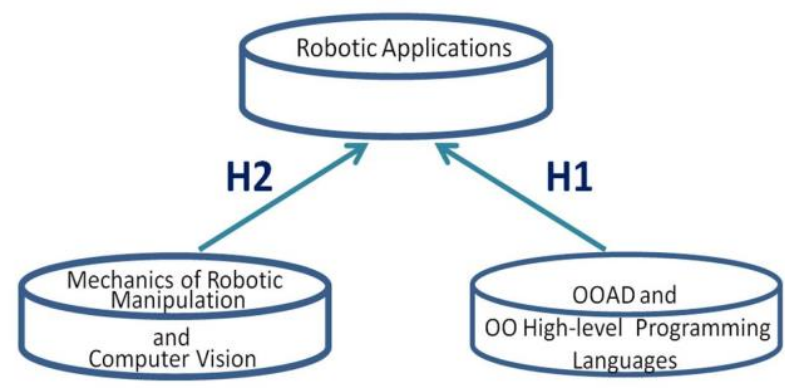

Fig.12. Theoretical Framework

As a result, Fig. 12 depicts the interrelationship between the independent variable Mechanics of Robotic Manipulation and Computer Vision and the dependent variable Robotic Applications. In the same way, Fig. 12 shows the interrelationship between the independent variable OOAD and OO High-level Programming Languages and the dependent variable Robotic Applications.

Consequently, this research introduces the following two nondirectional null hypotheses.

H1: OOAD and OO High-level Programming Languages do not support the analysis, design, and development of Robotic Applications.
H2: Mechanics of Robotic Manipulation and Computer Vision do not support the analysis, design, and development of Robotic Applications.

\section{RESEARCH QUESTIONS WITH HYPOTHESES}

Fig.13. illustrates the relationships of the Research Questions with Hypotheses. Accordingly, research question RQ1 leads to hypothesis H1 and research question RQ2 leads to hypothesis H2.

\begin{tabular}{|l|l|}
\hline \multicolumn{1}{|c|}{ Research Questions } & \multicolumn{1}{|c|}{ Hypotheses } \\
\hline $\begin{array}{l}\text { RQ1: Can undergraduate systems } \\
\text { engineering students apply OOAD } \\
\text { and OO High-level Programming } \\
\text { Languages to analyze, design, and } \\
\text { develop Robotic Applications? }\end{array}$ & $\begin{array}{l}\text { H1: OOAD and OO High-level } \\
\text { Programming Languages do not } \\
\text { support the analysis, design, and } \\
\text { development of Robotic } \\
\text { Applications. }\end{array}$ \\
\hline $\begin{array}{l}\text { RQ2: Can undergraduate systems } \\
\text { engineering students use } \\
\text { Mechanics of Robotic } \\
\text { Manipulation and Computer } \\
\text { Vision to analyze, design, and } \\
\text { develop Robotic Applications? }\end{array}$ & $\begin{array}{l}\text { H2: Mechanics of Robotic } \\
\text { Manipulation and Computer } \\
\text { Vision do not support the } \\
\text { analysis, design, and } \\
\text { development of Robotic } \\
\text { Applications. }\end{array}$ \\
\hline
\end{tabular}

Fig.13. Research Questions with Hypotheses

\section{Linking RoBotics With BIG DATA}

The combination of robotics with big data provides a wide-ranging of knowledge for robots. As long robots capture, accumulate, assign, manage, and perform analysis of big data repositories with high speed, the robot's intelligence will significantly increase. Therefore, big data plays an important role in robotics information management and decision making.

As depicted in Fig. 14, the suitable technologies available for managing big data environments [23, 24] for robotics are Hadoop Ecosystem and NoSQL Databases (Document Stored Database, Key-value Stored Database, Column-oriented Database, Object-oriented Database, and Graph Database).

Examples of some of the tools used by Hadoop Ecosystem are Apache Flume, Apache Sqoop, Apache Spark, Apache Hive, Apache Oozie, Apache Pig, Apache Mahout, Apache Hbase, and Apache Drill [23].

Likewise, MongoDB and CouchDB are examples of Document Stored Databases. In the same way, Amazon DynamoDB and Riak are examples of Key-value Stored Databases. Equally, BigTable, HBase, and Cassandra are examples of Column-oriented Databases. Similarly, db4o (database for objects) is an example of an Object-oriented Database. Finally, Neo4j is an example of a Graph Database [24]. 


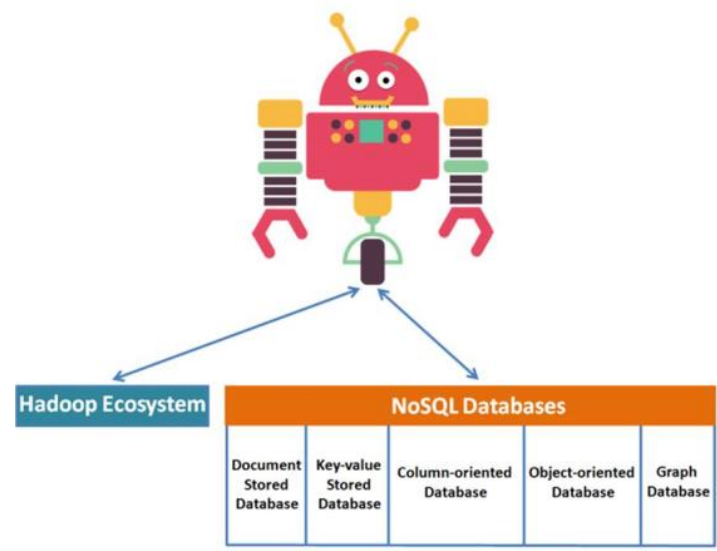

Fig.14. Linking Robotics with Big Data

\section{INTRODUCING CHATBOTS}

Since this paper is about AI and robotics, it is appropriate to introduce the idea of chatbots.

In a few words, chatbots are computer programs which interact with users using AI via normal sounding like conversations with the objective of answering questions and providing recommendations. Consequently, chatbots are also known as conversational robots.

However, with this kind of technology, developers build systems that mimic human conversations in order to make users believe that they are talking to real humans.

In that sense, chatbots have been developed for the retailing industry, universities, healthcare, airlines, sales and marketing, movie theaters, food services, hotels, and many other sectors.

In fact, chatbots represent a massive application of AI in the business world.

\section{Conclusion AND ReCOMmENDATIONS}

We have completed building an educational case study on teaching AI and robotics to undergraduate systems engineering students. Consequently, this research article was organized into the following sections: introduction, related work, describing the artificial intelligence and robotics' course, introducing the case study, resources of the course, robotic arm, theoretical framework, research questions with hypotheses, linking robotics with big data, introducing chatbots, and conclusion and recommendations.

As a result, hypothesis $\mathrm{H} 1$ stated that "OOAD and $\mathrm{OO}$ High-level Programming Languages do not support the analysis, design, and development of Robotic Applications" and hypothesis H2 denoted that "Mechanics of Robotic Manipulation and Computer Vision do not support the analysis, design, and development of Robotic Applications."

Therefore, the research approach used for testing the above hypotheses was the application of the developed case study. Consequently, it can be confirmed the rejection of hypothesis $\mathrm{H} 1$ and hypothesis $\mathrm{H} 2$.

Furthermore, the research question RQ1 stated the following interrogation: "Can undergraduate systems engineering students apply OOAD and OO High-level Programming Languages to analyze, design, and develop Robotic applications?" as part of the AI and robotics class.

Hence, RQ1 had a positive answer of "yes" because of the rejection of hypothesis $\mathrm{H} 1$.

Additionally, the research question RQ2 highlighted the following inquiry: "Can undergraduate systems engineering students use Mechanics of Robotic Manipulation and Computer Vision to analyze, design, and develop Robotic applications?" during the AI and robotics class.

Therefore, RQ2 had a positive answer of "yes" because of the rejection of hypothesis $\mathrm{H} 2$.

As a result, we have concluded that the rejection of $\mathrm{H} 1$ and $\mathrm{H} 2$ helped to find the answers for RQ1 and RQ2.

Consequently, integrating AI with robotics formed the ground rules of AI-robotics Ecosystem having robots connected with big data platforms such as Hadoop Ecosystem and NoSQL Databases embedded within a context of AI.

Accordingly, the following ideas have emerged as a base for additional research: identify machine learning and deep learning models for robotics, develop a hightech strategy addressing the support of AI-robotics Ecosystem using microservices and containers technologies, apply collaborative mobile learning (MLearning) technology [25] to AI and robotics learning, design a computer architecture for robotics based on NoSQL Databases, implement a multimedia education program [26] to support the AI-robotics Ecosystem, design a robotic-oriented architecture based on Hadoop Ecosystem, study the application of social networks [27] to the AI-robotics Ecosystem's collaborative learning environment, create a chatbot to provide advice to companies and senior executives in corporate strategy development and implementation, create a chatbot to provide guidance to Alzheimer's disease patients' families, friends, and caregivers, create a chatbot to provide guidance to Tuberculosis' disease patients' families, friends, and caregivers, and develop a full lifecycle methodology for creating and/or documenting chatbot systems.

\section{ACKNOWLEDGMENT}

We'd like to thank Aurilú Rivas Moreno and Jhosenn Camacho for editing the manuscript of this paper. Similarly, we'd like to give many thanks to the following senior systems engineering students for participating in this research: Edgar Alvarado, Mariana Camejo, Darelyssmar Castro, Alexander Paredes, and Oscar Perdomo. Thank you so much!

\section{REFERENCES}

[1] Tutorialspoint, Artificial Intelligence: Intelligence Systems, Tutorials Point (I) Pvt. Ltd., 2015. 
[2] M.A. Boden, "Creativity and artificial intelligence," Artificial Intelligence, vol. 103, pp. 347-356, 1998.

[3] A. Chella, L. Iocchi, I. Macaluso, and D. Nardi, "Artificial Intelligence and Robotics," Contributi Scientifici, Anno III, No 1/2, Marzo-Giugno, 2006.

[4] Y. Korem, Robotics for Engineers, McGraw-Hill Book Company, 1985.

[5] F. Ingrand and M. Ghallab, "Robotics and Artificial Intelligence: a Perspective on Deliberation Functions," AI Communications, 27(1), pp. 63-80, 2014.

[6] T. Niemueller and S. Widyadharma, "Artificial Intelligence - An Introduction to Robotics," In Proseminar of Artificial Intelligence at the RWTH Aachen, pp. 1-14, 2003.

[7] B. Coppin, Artificial intelligence illuminated, Jones and Bartlett Publishers, Inc., 2004.

[8] G. Zabala, Robótica: Guía Teórica y Práctica, Gradi S.A., 2007.

[9] F. Klassner, "A Case Study of LEGO Mindstorms'TM Suitability for Artificial Intelligence and Robotics Courses at the College Level," SIGCSE'02, February 27-March 3, pp. 8-12, 2002,

[10] A. Eguchi, "Educational Robotics for Promoting 21st Century Skills," Journal of Automation, Mobile Robotics \& Intelligent Systems, vol. 8, 1, pp. 5-11, 2014.

[11] D. Alimisis, "Educational robotics: Open questions and new challenges," Themes in Science \& Technology Education, 6(1), pp. 63-71, 2013.

[12] P. Robinson, "Robotics education and training: a strategy for development," Industrial Robot, vol. 23 issue: 2, pp. 4-11, 1996.

[13] D. Wong, R. Zink, and S. Koenig, "Teaching Artificial Intelligence and Robotics Via Games (Abstract)," In Proceedings of the Twenty-Fourth AAAI Conference on Artificial Intelligence (AAAI-10), pp. 1917-1918, 2010.

[14] P.M. Taylor, "University education in robotics and advanced automation: a UK perspective," Industrial Robot, vol. 23, issue: 2, pp.16-20, 1996.

[15] K.B.M. Noor, "Case Study: A Strategic Research Methodology," American Journal of Applied Sciences, 5 (11), pp. 1602-1604, 2008.

[16] L.E. Alvarez-Dionisi and G. Tapia de Vidal, "Case Studies: Developing Financial Mathematics' Systems," Social Economic Debates, 6(1), pp. 34-47, 2017.

[17] Z. Zainal, "Case study as a research method," Jurnal Kemanusiaan. Bil. 9, pp. 1-6, Jun 2007.

[18] S.J. Russell and P. Norvig, Artificial Intelligence: A Modern Approach Third Edition, Pearson Education, Inc., 2010.

[19] J.J. Craig, Introduction to Robotics, Pearson Education, Inc., 2005.

[20] M.A. Peshkin, Robotics Manipulation Strategies, Prentice-Hall, Inc., 1990.

[21] Universal Robots Academy, Training Modules, https://www.universal-robots.com/academy/, 2017.

[22] J. Angeles, Fundamentals of Robotic Mechanical Systems: Theory, Methods, and Algorithms, 3er Edition, Springer Science+Business Media, LLC, 2007.

[23] L.E. Alvarez-Dionisi, "Toward Grasping the Dynamic Concept of Big Data," I.J. Information Technology and Computer Science, vol. 8, 7, pp. 8-15, 2016.

[24] L.E. Alvarez-Dionisi, "Envisioning Skills for Adopting, Managing, and Implementing Big Data Technology in the $21^{\text {st }}$ Century," I.J. Information Technology and Computer Science, vol. 9, 1, pp. 18-25, 2017.
[25] S.T. Njenga, R.O. Oboko, E.I. Omwenga, and E.M. Maina "Use of Intelligent Agents in Collaborative M-Learning: Case of Facilitating Group Learner Interactions," I.J. Modern Education and Computer Science, vol. 10, pp. 18-28, 2017.

[26] Z. Kotevski and I. Tasevska, "Evaluating the Potentials of Educational Systems to Advance Implementing Multimedia Technologies," I.J. Modern Education and Computer Science, vol. 1, pp. 26-35, 2017.

[27] A.A. Alkhathlan and A.A. Al-Daraiseh, "An Analytical Study of the Use of Social Networks for Collaborative Learning in Higher Education," I.J. Modern Education and Computer Science, vol. 2, pp. 1-13, 2017.

\section{Authors' Profiles}

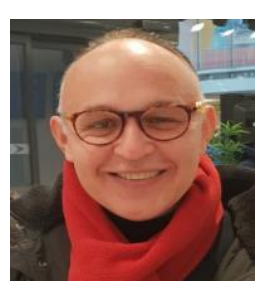

Dr. Luis Emilio Alvarez-Dionisi is a professor of AI, machine learning, and deep learning. He is an international management consultant with extensive experience working with chief executive officers, boards of directors and senior management in Fortune 500 companies. $\mathrm{He}$ has advised numerous organizations worldwide, including Intel, IBM, Merck, Chevron, Isuzu, Smiths Detection, the Beijing 2008 Olympic Games, and the Government of Singapore Investment Corporation (GIC) on project, program, and portfolio management. Alvarez-Dionisi's research work focuses on global project management trends, agile project management, AI, cybersecurity culture, chatbots for business, engineering robotics, big data applications, IT governance, and medical information systems.

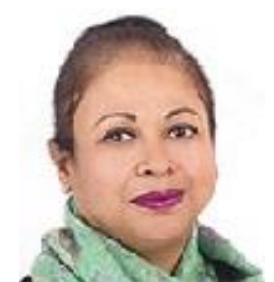

Dr. Mitali Mittra is a professor of management and director of the Kingston/RANEPA MBA program and the master's degree in project management at RANEPA. Likewise, Mittra teaches international \& strategic marketing, research methodology, and management consultancy. She used to be the head of marketing research of the British Council. Similarly, she was the marketing and communication director for TMI (a well-known UK consulting organization). Mittra has more than 15 years of working experience in marketing with different international companies. Currently, she runs her own consulting firm providing services in the areas of marketing, strategy, and communication.

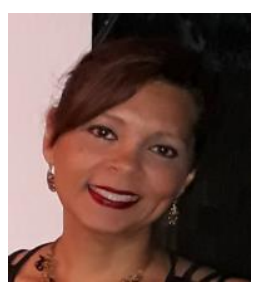

Rosbelia Balza, MSc, is a system engineer with a master's degree in management science. Currently, Balza is a professor and director of the School of Systems Engineering at the Polytechnic University Institute "Santiago Mariño" in Barinas, Venezuela. She teaches Java programming, design and evaluation of projects, data structure, databases, systems analysis \& design, operating systems, computing auditing, $\mathrm{C}++$ and PHP programming, and management information systems. 
How to cite this paper: Luis Emilio Alvarez-Dionisi, Mitali Mittra, Rosbelia Balza, "Teaching Artificial Intelligence and Robotics to Undergraduate Systems Engineering Students", International Journal of Modern Education and Computer Science(IJMECS), Vol.11, No.7, pp. 54-63, 2019.DOI: 10.5815/ijmecs.2019.07.06 